\title{
A Composite Framework of Co-located Asymmetric Virtual Reality
}

\author{
KAITLYN M. OUVERSON, Iowa State University, USA \\ STEPHEN B. GILBERT, Iowa State University, USA
}

\begin{abstract}
As the variety of possible interactions with virtual reality (VR) continues to expand, researchers need a way to relate these interactions to users' needs and goals in ways that advance understanding. Existing efforts have focused mainly on the symmetric use of technology, which excludes a rising form of interaction known as asymmetric VR, in which co-located participants use different interfaces to interact with a shared environment. There must be a clear path to creating asymmetric VR systems that are rooted in previous work from several fields, as these systems have use cases in education, hybrid reality teams (using VR and other technologies to interact online and face to face), accessibility, as well as entertainment. Currently, there is no systematic way to characterize 1) how a system may be asymmetric, 2) how the different mediation technology and affordances within asymmetric VR support (or do not support) users' goals, and 3) the relationships and collaborative capabilities between users of these different technologies. In this paper, the authors use a scoping review to explore relevant conceptual frameworks for asymmetric interaction, mediation technology, and computer supported cooperative work to clarify the dimensions of asymmetry and synthesize the literature into a Composite framework for Asymmetric VR (CAVR). The paper concludes with suggestions of ways to test and expand the framework in order to guide future research as it identifies the most-beneficial interaction paradigms for co-located asymmetric VR.
\end{abstract}

CCS Concepts: - Human-centered computing $\rightarrow$ Collaborative and social computing $\rightarrow$ Collaborative and social computing theory, concepts and paradigms $\rightarrow$ Computer supported cooperative work $\bullet$ Human-centered computing $\rightarrow \mathrm{HCI} \rightarrow$ Interaction paradigms $\rightarrow$ Virtual reality; Collaborative interaction; Mixed / augmented reality

KEYWORDS: Extended reality (XR); Mixed reality (MR); Conceptual Frameworks; Asymmetric VR; Workspace Awareness; Collaboration

ACM Reference format:

Kaitlyn M. Ouverson and Stephen B. Gilbert. 2021. A Composite Framework of Co-located Asymmetric Virtual Reality. In PACM on Human Computer Interaction, Vol. 5, No. CSCW1, Article 5, April 2021. New York, NY, USA. 20 pages. https://doi.org/10.1145/3449079

\section{INTRODUCTION}

Asymmetry, as it relates to technology, has been defined as "the capacity of individuals in a group to have different means to visualize and interact with virtual content" [16:127]. However, as it relates to virtual reality (VR), asymmetry is more than just the visual and interactive aspects. If VR

Author's addresses: K.M. Ouverson, Iowa State University, Human Computer Interaction Program, IA, USA, kmo@iastate.edu; S.B. Gilbert, Iowa State University, Department of Industrial and Manufacturing Engineering, IA, USA, gilbert@iastate.edu

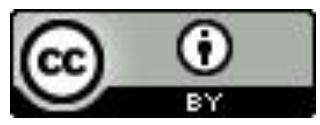

This work is licensed under a Creative Commons Attribution International 4.0 License.

2573-0142/2021/April - Art5. https://doi.org/10.1145/3449079

PACM on Human-Computer Interaction, Vol. 5, No. CSCW1, Article 5, Publication date: April 2021. 
is to be the next teleconferencing medium [14], and if the technology is to be more accessible, the insights of multiple fields that have studied asymmetry must be brought together as guidance. That is not to say that asymmetry should be viewed as a challenge to overcome. As previous authors have concurred, asymmetry is simply present in social interaction, and in some cases, it can even be beneficial to a shared task $[3,35,51]$. Still, there must be a clear path for understanding and leveraging asymmetric use of co-located multi-user VR, as these mixed-reality spaces are vital to the acceptance and accessibility of VR. Following a scoping review of the literature, this paper synthesizes 18 conceptual works into one Composite framework for Asymmetric VR (CAVR), including a reframed definition of the ways in which asymmetry can occur and an acknowledgment of the context surrounding asymmetric VR use.

Up to this point, the underlying topic areas related to asymmetric VR, including ludology (the study of video games), social psychology, CSCW, education technology, and VR, have remained disparate. The ludology perspective [22] considers asymmetry as a way of facilitating certain kinds of tasks. For example, if a team needs to change a design that is meant for use in a headmounted display (HMD), using VR may be the most straightforward way to test the environment, while a desktop application (and the granular control afforded by mouse and keyboard) may be most suited for making edits. If notetaking is necessary to the workflow, the task is poorly supported for an HMD-user [24,38] but well-suited to a desktop-user. Ludology insights would suggest that the design task can be described as having sequential timing, meaning the two users take turns, while the notetaking task has expectant timing, in which the desktop user waits for the HMD user's signal to take action [22]. Work that applies these insights to education technology suggests that notetaking is supported by high asymmetry, whereas the design task would necessitate a medium asymmetry environment [49].

More information is found in social psychology literature [46]. In a mixed-reality space, such as exists during the use of asymmetric VR, certain information can be hidden from different group members. When using an HMD, a designer would not see the co-located others' locations or deictic references. When not in the HMD, the proper depth and immersion of the environment is hidden information. If teams are to use asymmetric VR, it will be essential to make hidden information visible to all members, especially when it is relevant to group decision making [46]. While the information needed for each role and how to provide such information is not available in this discipline's literature, some hints are scattered in the field of CSCW.

CSCW offers several relevant theories (enhancement technology and workspace awareness, for example). Looking at the VR design task from an enhancement technology perspective [42], the existing technology in the environment could be leveraged to supply extra information that facilitates conversation around the virtual design. To understand more about what kinds of information (in addition to verbal conversation information) are necessary for fostering a functional group interaction in the space, one can examine workspace awareness literature [19]. A team's needs for establishing workspace awareness are clearly outlined, but the application of workspace awareness to asymmetric VR is not.

Innovation in CSCW technology is an ever-continuing stream, and for research to keep pace, flexible yet precise models are necessary. Models in this field have begun adapting to include more nuanced topics. For instance, Lee and Paine [32:184] use the term coordinated action to recognize the growing interest of studying the activity of two or more people as they work "towards a particular goal through one or more overlapping fields of action." By redefining "work," they include goal-directed action that is traditionally excluded from the research focus of CSCW. Carefully established frameworks improve the ease with which complex topics, such as 
collaboration and asymmetric VR, can be researched. Such frameworks facilitate researchers' interpretation of the data so that the best possible conclusions may be drawn, for example, by guiding the selection of variables to be manipulated, measured, and controlled. This paper seeks to do just that, bringing together the various existing frameworks as part of an effort to create an integrative, composite framework: CAVR.

\subsection{Background: Need for a framework of asymmetric VR}

As extended reality (XR), a category including VR, AR (augmented reality), and MR (mixed reality), has gained popularity, the focus of research has shifted from getting the hardware to work to understanding the consequent human interactions [14]. As explained by Olsson et al. [42], much research has been done to understand what best supports, or enables, the interactions between distantly-located humans, but relatively little work has focused on how technology can enhance co-located interaction. XR is no exception to this trend, and computer-mediated support for co-located interaction during $\mathrm{HMD}$ use is essential to the widespread adoption of the technology [18]. The experience of VR can be an isolating one, so HMD-users (and their onlookers) need technology mediation as they interact with one another. The solution is not as simple as "just put each collaborator in their own HMD," or "just show collaborators a monitor with what the HMD-user is seeing." Each of these options oversimplifies the collaboration needs and dynamics [3,33-35,51]. As such, the authors propose a focus on harnessing the abilities of asymmetric design to foster engaging VR experiences.

Asymmetric VR, a form of VR interaction in which co-located users access the same virtual environment using different kinds of technology, has gained popularity within research since the phrase was coined in 2012 to describe virtual telepresence [47]. The term and ensuing research draw from earlier studies describing the asymmetry of virtual movement [45] and media spaces [23,51]. However, in colloquial use on discussion forums such as Reddit, asymmetric VR specifically describes co-located use of a mix of technology, while the remote use of asymmetric technology is described as cross-platform multiplayer VR. In VR research, the focus has traditionally stayed closer to symmetric and single-device use. For example, Milgram and Kishino [33] describe the continuum of technology-enabled environments between physical reality on one end of the spectrum and VR on the other end. While the virtuality continuum describes the technology quite well, Benford and colleagues [3] find another axis, which relates to the way in which objects are either brought to the user or the user is brought to them, is necessary to better describe group use of the technology.

Even with VR frameworks extended to include support for collaborative uses, Ens et al. [14] note more work is needed to merge CSCW groupware and VR into a common conceptualization of MR for coordinated action. After their extensive review of MR collaboration research, Ens and colleagues [14] conclude that the current methods of categorizing and understanding cooperative systems are not sufficient for use as frameworks for the study of MR. Similarly, their review highlights gaps in the present understanding of asymmetry and of asymmetric VR. While asymmetric VR research is present in the last 30 years, just over three-quarters of the papers on asymmetric use of XR technology were published after 2010, and the focus has largely concerned remote asymmetry (or, cross-platform multiplayer VR) which combines AR and VR to allow experts to assist novice users remotely [14].

Because asymmetry necessitates at least two individuals, similar to team interactions, organizational psychology concepts of teamwork dynamics, team composition, and team emergent states are relevant. Teamwork dynamics include the groups' interactions (including 
dependency $[21,22,37]$ ) and the support of these interactions by the technology (asymmetric VR). Also, team composition includes factors that may impact the choice to use asymmetric VR - such as the team's accessibility needs, power balance (as influenced by personality traits and expertise [54]), and multidisciplinary nature (or heterogeneous roles). In team research, emerging states, including the group's attitudes, behaviors, and their thoughts and understanding of the situation, mediate collaboration outcomes [54]. In asymmetric VR, each team member's workspace awareness will likely be influenced by the level of asymmetry in the experience. Additionally, it seems reasonable to assume that different levels and kinds of asymmetry may correlate with collaboration outcomes, prosocial behaviors, and team member rapport, as these are associated with interdependence $[4,5,27,44]$, which is fostered by asymmetry [21].

In 1988, when CSCW was a nascent field fueled by the introduction of the internet and email in corporate settings, Johansen [26] organized a matrix of time and place to mobilize the field toward a common goal. This matrix has become ubiquitous in the field [32] and is used to classify electronic meetings as "Asynchronous" or "Synchronous" and "Local (Co-located)" or "Distributed". While a useful tool, the organization of electronically-mediated communication on these two axes hides the complexity of technology use related to asymmetry. For instance, while the synchronous asymmetric use of VR in a single physical space (e.g., one person in an HMD and two people who are not) is technically co-located use, the fracturing of both the environment surrounding the generation of an action and that accompanying the deciphering of said action raises novel design considerations [30,34,36]. This fracturing means asymmetric-VR-using groups do not receive the same benefits as synchronous, co-located groups using other technology during their coordinated action, despite the suggestion of Johansen's matrix [26].

Asymmetric VR may be a useful tool for businesses as they continue to embrace virtual teams. Even as hardware becomes cheaper and the coordination of multiple HMD-users in a single physical space is optimized, it is unlikely that all organizations will acquire sufficient headsets for everyone. It is also highly probable that certain tasks will require virtual and physical artifacts - as shown in earlier studies of cooperative work [28] - or that co-located teams will interact with other teams that are co-located in separate offices. To support the heterogeneous roles of teams in these mixed-reality spaces, clear recommendations for optimizing the collaborative experience within the space are necessary but have yet to be established.

For the general public, asymmetric VR video games are a popular first experience with VR technology. In 2015, before the Oculus Rift CV1 came to market, Steel Crate Games released "Keep Talking and Nobody Explodes," a game that leverages the asymmetries induced by the presence of the HMD to create a compelling experience (rated 10/10 on Steam, a popular game platform for VR) [48]. Keep Talking is one of the first games someone may have purchased for showing VR to friends; rather than passively waiting for a turn with the HMD, the spectators found themselves engaged in the experience. Outside of video games, HMD-induced asymmetry has seen attention in collaborative 3D design [16], data analysis [12], and public experiences such as museums $[15,17]$. Similar to the idea of "blended classrooms" [7], asymmetric VR may also find application in HMD-based training and classroom education [49]. In each of these situations, researchers and designers need language to describe how asymmetric experiences differ.

One area of application that has not seen much attention in VR, especially in asymmetric VR literature, is the facilitation of accessibility to the virtual (or mixed-reality, in asymmetric set-ups) space. Research on VR with populations such as older adults and people with disabilities is limited and typically focuses on using 2D screens and projected VR rather than consumer HMDs (although exceptions exist [6,9]). Tools to increase the accessibility of 6DOF (6 degrees-of- 
freedom) devices for people who are confined to beds, use wheelchairs, or have limited use of their arms (e.g., have one arm) are beginning to come to market (such as WalkinVR Driver [52]). Asymmetric VR may offer alternative or complementary ways to access the virtual space by people who choose not to don an HMD for a variety of reasons yet desire a connection to others experiencing VR. To understand the impacts of asymmetric interaction on groups that are diverse in a multitude of ways, including in their accessibility needs and available tools, researchers' effort needs to centralize around an integrative framework that can offer a common language for asymmetric VR experiences.

\section{METHODS}

One powerful method of organizing research efforts is the literature review. Scoping reviews can be used to examine and clarify key concepts, creating comprehensive definitions of an area, rather than a systematic answer to a question [40]. Much like a systematic literature review, scoping reviews follow a protocol to exhaustively identify and extract data from a corpus of published papers [40]. For the present work, the Scopus citation database was used to identify empirical work on asymmetric VR. The terms used in the initial search were a VR term ("Virtual Reality" or "VR" or "Mixed Reality" or "Head-Mounted Display" or "HMD") AND either a collaboration term ("collaborative" or "collaboration" or "asymmetric technology" or "cooperative" or "cooperation" or "bystander" or "multiple head-mounted displays" or "multiple HMDs" or "multi-user" or "multiple user") OR a location term ("co-located" or "collocated" or "shared space" or "same space”).

The initial 257 papers were evaluated against a set of inclusion criteria, namely collecting only publications in which: 1) studies included at least one head-mounted virtual reality device, 2) the data were collected at the level of the individual, and 3) papers were published between 2014 and January 2020 (when the search happened), with the lower-bound corresponding to the release of the Oculus Rift Development Kit, which represents a dramatic increase in quality and affordability of VR HMDs. This process resulted in a total of 109 papers.

The advancement in technology which spurred the time limit of the initial search was itself afforded by prior research, which could mean important research was missed in the initial search. However, as the intention was to use these articles to find foundational works from which the recent wave of VR popularity benefits, the references of the 109 papers were subsequently examined for relevant work both within and beyond the initial time limitation. As a result, an additional 91 articles were identified for a total of 200 articles on co-located, asymmetric VR.

Rather than summarizing the themes embodied in the results of these 200 papers, as may be more typical in a scoping review, the authors' goal was to identify the range of underlying conceptual frameworks spanned by the corpus of 200. The relevant conceptual frameworks underlying these 200 academic works were identified, either cited by the papers themselves or related to multiple papers. This resulting collection of 18 "framework papers" represents ideas from CSCW [2,10,14,19,26,32,42,51], VR [3,30,39], ludology [21,22], group decision making [46], organizational psychology [37,54], economics [1], and education technology literature [49]. These papers were then used to develop CAVR, a framework that draws on the multiple frameworks found.

Section 3 describes the key frameworks of the 18 theory papers. Section 4 presents CAVR and how it is derived from these frameworks. Section 5 discusses the ramifications of CAVR and describes future work. 


\section{THE RANGE OF RELEVANT CONCEPTUAL FRAMEWORKS}

This section presents the conceptual frameworks explored in the 18 framework papers which subsequently led to CAVR. The work is grouped into the following clusters: 3.1 ) asymmetry of information, 3.2) asymmetry in video games and media spaces, 3.3) asymmetric awareness of others and the environment, 3.4) interdependence and timing within asymmetric systems.

\subsection{Asymmetry of Information}

The idea of asymmetry has long been examined in contexts beyond technology. The most prominent of these surrounds the asymmetry of information $[1,46]$. While information asymmetry was not directly cited in the corpus, CAVR identifies this research thread as important to understanding the group use of technology as present in asymmetric VR.

In the 1960s, economist Kenneth Arrow described the disparities created by information differences between buyers and sellers, particularly related to the uncertainties in the medical field [1]. His article applied mathematics to the handling of information and concepts such as "trust" that were previously difficult to explore systematically [20]. In the 1980s, small groups research focused on the asymmetric knowledge of a group of 2-5 individuals. In the Hidden Profile Paradigm [46], members of a group are given access to information about fictitious candidates for a job and asked to choose the best one. Each group member has access to exclusive information about the candidate as well as information shared by all members of the group. Most groups focus only on shared information, but the only way to choose the best candidate is to consider individual members' exclusive information. One of the main goals of this research task is to deduce the conditions under which members did or did not share the hidden information, and the influencing factors often included group dynamics such as rapport, incentives, and power [46]. Because computer-mediated communication, including asymmetric VR, can affect these group dynamics significantly, it is useful to consider that asymmetric technology may be more important to specific group members, depending on the information they possess.

\subsection{Asymmetry in Media Spaces and Video Games}

Within the 200-paper corpus, asymmetry was typically identified through either a ludology (video games) or a media spaces lens. Through the media spaces lens, fractured ecologies and asymmetric perceptual access were influential to research and development of asymmetric VR experiences. Fractured ecologies, or differences in the immediate contexts of action generation and comprehension $[23,34]$, introduce types of asymmetry related to, but different from, information asymmetry. Referred to as the asymmetry of perceptual access [53], this type of asymmetry arises for two reasons. First, even as computer-based solutions move into a workplace, physical artifacts from paper to medical tools are necessary (or perceived as such) to the work being done [33,53]. Second, computer use may occur from a remote location or may need to be conducted in a separate room [53]. As VR continues to grow in consumer and industrial popularity, it follows that some physical artifacts will continue to be necessary, just as was true for the introduction of computers into long-established areas of work. Therefore, fractures in the ecological basis of actions for HMD-users and external users of asymmetric VR are impactful to the users' experiences, regardless of their co-presence in the physical location.

Many people's first VR experience arises through video games. Video game designers are often free to implement novel paradigms within their simulated environments, meaning the interactions 
and interfaces introduced in video games can offer insights to emergent technology trends (as in previous work examining human cooperation with autonomous agents through video game analysis [50]). By designing a game that leverages the information, context, and task differences between players, creators can make specific choices to increase the chances that any individual player values their game experience, e.g., by presenting play styles. In doing so, asymmetric games both cater to differences between players, such as skill level or amount of (pre-existing or gameprompted) available information and create multi-faceted experiences that promote tightly coupled social interaction through interdependence [21]. Asymmetric experiences for other kinds of coordinated action may similarly impact collaboration outcomes, and as such, a more thorough understanding of asymmetric VR results from incorporating scoping review insights from ludology.

While the ludology and media spaces frameworks that focused specifically on asymmetric technology did not include VR, the two frameworks are critical to the derivation of CAVR. According to Harris et al., the infusion of asymmetry into video game mechanics may occur as an asymmetry of ability, challenge, interface, information, investment, or goal/responsibility [22]. Harris and colleagues also extend the Mechanics, Dynamics, and Aesthetics (MDA) framework [25] to better accommodate asymmetric multiplayer game design. The MDA framework is a combination of understanding how the design choices in a game (Mechanics) impact a player's experiences in a game (Dynamics) and how those experiences impact how players feel about that game (Aesthetics). While the designer has control over the dynamics and mechanics of a game, players can have different reactions to the same game, resulting in a variety of aesthetics.

While Harris and colleagues focus on the interaction dynamics of an asymmetric video game [22], Voida and colleagues [51] examine the asymmetry of characteristics of media spaces, including traditional and social media. Using this lens, Voida et al. delineate asymmetry as including fidelity, place, participation, media, engagement, and benefit [51]. Table 1 summarizes each mechanic of asymmetry based on games [22] and forms of asymmetry based on media spaces [51]. Of interest to the present research, these definitions do not naturally converge to form a complete understanding of asymmetry. Asymmetric VR, as described in Section 1.1, is a form of VR interaction in which co-located users access the same virtual environment using different kinds of technology, such as a desktop monitor with a keyboard and mouse or a touchscreen device. Consequently, asymmetric VR necessarily facilitates coordinated action, including cooperation, collaboration, and competition, between two or more individuals. The asymmetry in asymmetric VR refers to the differences between the experiences of these multiple actors.

One of the aims of CAVR is to offer consistent terminology to describe the experiences and effects of asymmetric VR. To better align these previous works for use in describing Asymmetric VR, the following process was observed: 1) three independent reviewers extracted the definitions of asymmetry from the Voida et al. [51] and Harris et al. [22] papers, 2) the reviewers worked independently to group the given definitions in a way that reflected their relationship to asymmetric VR use, 3) the reviewers independently developed definitions of the groupings, 4) the reviewers met to discuss their findings to promote consensus around dimensions which were not redundant yet exhaustively descriptive of the concept, and 5) the final definitions were contextualized in light of the scoping review. This process, along with the scoping review, aided in the definition of the dimensions of CAVR. 
Table 1. Definitions of game design mechanics [22] and forms of asymmetry [51].

\begin{tabular}{|c|c|c|c|}
\hline Mechanics $^{\top}$ & Definition & Forms ${ }^{\mp}$ & Definition \\
\hline Ability & $\begin{array}{l}\text { Users have different actions } \\
\text { they can take. }\end{array}$ & Fidelity & $\begin{array}{l}\text { There can be different quality } \\
\text { and frequencies of information. }\end{array}$ \\
\hline Challenge & $\begin{array}{l}\text { Users face different kinds of } \\
\text { obstacles. }\end{array}$ & Place & $\begin{array}{l}\text { The context of use of a system } \\
\text { influences the expectations. }\end{array}$ \\
\hline Interface & $\begin{array}{l}\text { Users have different ways of } \\
\text { engaging with (input and } \\
\text { output) the system. }\end{array}$ & Participation & $\begin{array}{l}\text { Users have different roles and } \\
\text { levels of control within the } \\
\text { system. }\end{array}$ \\
\hline Information & $\begin{array}{l}\text { Users receive different kinds of } \\
\text { information about the system } \\
\text { and one-another. }\end{array}$ & Media & $\begin{array}{l}\text { Users share different kinds of } \\
\text { information. }\end{array}$ \\
\hline Investment & $\begin{array}{l}\text { Users dedicate different } \\
\text { amounts of effort and time to } \\
\text { their roles. }\end{array}$ & Engagement & $\begin{array}{l}\text { Users give different levels of } \\
\text { attention or focus to the } \\
\text { system. }\end{array}$ \\
\hline Goals & $\begin{array}{l}\text { Users seek different outcomes } \\
\text { from the system. }\end{array}$ & Benefit & $\begin{array}{l}\text { The system offers different } \\
\text { solutions to user's needs. }\end{array}$ \\
\hline
\end{tabular}

†Adapted from [22], †Adapted from [51]

\subsection{Asymmetric awareness of others and the environment}

This section introduces results from the scoping review regarding how collaborating users maintain awareness of both the state of the coordinated action and the actions of their colleagues in a shared workspace. In order for asymmetry to foster collaboration or cooperation, those involved in the experience must have access to information about what is happening, by whom, and where the action is occurring in the mixed-reality space [19]. As such, three existing frameworks - workspace awareness, channels of communication, and the framework of enhancement technology - are introduced in this section.

When designing a system to support coordinated action in the presence of asymmetry, including for co-located interactions, one should consider how people use a typical workspace environment. In 2002, Gutwin and Greenberg [19] published a framework of workspace awareness based on the ways in which small groups of two to five people use a shared workspace. Users of groupware applications need to keep track of, gather, and use workspace awareness in collaboration. The framework points to how each of these is accomplished and how they can break down - especially the failures to 1) gather necessary information and 2) understand what that gathered information means. According to the framework, groups need to answer questions of "Who," "What," "Where," "When," and "How," about the workspace and the objects and people who occupy it.

While Gutwin and Greenberg [19] derived their understanding of awareness from co-located use of physical work-surfaces, the theory is useful when considering any workspaces through which small groups interact. Among other workspaces, the framework has seen application to telecommunication [19] and video games [55]. For this reason, the framework of workspace awareness serves a foundational role in understanding how co-located groups interact with a single virtual space using asymmetric VR. 
A primary way of gathering workspace information is through communication, either 1) consequential of others' actions in the environment, 2) as feedthrough from artifacts, or 3) through intentional communication [19]. Kraus and Kibsgaard [30] recognize four communication channels through which consequential, feedthrough, and intentional communication may pass: auditory, visual, media-based (or pre-recorded), and a collective "other" category, including channels that are atypical candidates for technological mediation (such as olfactory and haptic). In this context, mediation refers to the mediation of communication, which Kraus and Kibsgaard posit is broken into three levels (unmediated, transparent mediation, and explicit mediation) that hold a negative relationship to the level of co-presence relative to costs of implementation. Considering this, they note that explicit mediation, though it produces the lowest levels of copresence, may result in better performance and team outcomes than transparent mediation implemented at the same cost [30]. In other words, while co-presence is higher in unmediated and transparently mediated environments, mediation that is easily identifiable by participants (explicit mediation) can produce better collaboration than half-implemented transparent mediation. This framework of communication channels also informs the use of asymmetric VR, which could be framed as a form of explicit mediation.

Olsson et al. [42] suggest a need to study further the co-located use of technology in groups (which Kraus and Kibsgaard [30] choose to avoid, classifying these as single-user experiences instead). When considering asymmetric VR, one can understand how the systems support or discourage interaction within the co-located group using the Framework of Enhancement Technology [42]. Following a scoping review of 92 studies on co-located technology use, this framework consists of nine design objective categories and eight design approach categories [42]. Among the identified design objectives is the need to accommodate asymmetric interaction, whether group members experience asymmetry in the type of device or in its user support, making this a key building block to CAVR.

The Framework of Enhancement Technology [42] describes three technology roles that aim to enhance co-located interaction rather than simply enable it. First, the role of inviting interaction pertains to technology that motivates new encounters among co-located others by signaling potential social interaction partners' interests and availability. Second, technology that facilitates interaction supports equitable interaction dynamics for co-located others with whom you would otherwise interact. Third, that which encourages interaction incentivizes people to either start or maintain their ongoing interactions, providing opportunities and stimulating action through appropriate affordances. Curiously, the authors of this 2019 review did not consider any literature on VR while creating their framework. While their search did not include VR applications, it seems reasonable to link work on enabling interaction with technologies from smartwatches to smartphones to the study of enabling co-located interaction among immersed and non-immersed users of VR.

However, due to this lack of representation in the review, it is not clear how asymmetric VR is best represented within the Framework of Enhancement Technology. More work is needed to understand relationships between the asymmetry configuration and the specific interaction role (as determined by the Olsson et al.'s framework [42]) of an asymmetric VR experience. By altering the mediation to support the asymmetric experience, even for coordinated action involving a nonimmersed user and an immersed user, positive group outcomes may be achieved [30]. And, by focusing on supplying the information required for maintaining workspace awareness [19] through the available communication channels [30], distinguishing the missing components of complex group interaction in MR, namely the unique requirements for workspace awareness in asymmetric VR, can begin.

PACM on Human-Computer Interaction, Vol. 5, No. CSCW1, Article 5, Publication date: April 2021. 


\subsection{Interdependence and Timing}

The current scoping review revealed one conceptual paper on asymmetric VR. Thomsen et al. [49] offer an existing characterization of asymmetry that includes an extension of the Harris et al. [22] asymmetric video game design elements to VR. However, the Thomsen et al. taxonomy focuses more on interaction design, including the timing of actions and direction of dependence within an asymmetric system rather than offering a broader view that includes the multitude of ways to experience asymmetry within VR. Even still, this theory is critical and contributes to the CAVR dimension of team interdependence.

Under conditions of cooperation, the direction of dependence can be classified as an operationalization of the team definition, commonly stated as two or more people who work, more or less dependently, to accomplish a shared goal [54]. These directions include mirrored, unidirectional, and bidirectional dependence [22]. Mirrored dependence is typical in traditional cooperative games, wherein Player A and Player B rely on each other in identical ways. In unidirectional dependence, Player B does not reciprocate Player A's reliance. Bidirectional dependence is similar to mirrored dependence, except that the two players' reliance takes a different form for each player, as is expected for teamwork involving multiple roles and collaborative interactions [54].

A key factor of interdependence (or dependence) is the timing of actions. Action timing [22,49] is organized into five types. Coincident actions (1) occur simultaneously, while sequential/disjoint actions (2) occur in sequence with no required amount of time between them. Similar to coincident, concurrent actions (3) occur simultaneously, but they require coordination in a way that is not linked to the time variable. Actions that require no coordination between actors are asynchronous (4). Finally, actions are expectant (5) when they require one member to anticipate the execution of another's action (or that other's signal) before performing their own action.

Whereas timing arose within ludology, researchers from CSCW also deal in the time context of actions and tasks. Originally dichotomized as synchronous or asynchronous in Johansen's [26] matrix, more recent work [13,32] extends the matrix to include concurrent synchronized, serial, mixed, and unsynchronized timing. Mapping the work of Dix et al. [13] to that of Harris et al. [22], these can be defined as follows: Concurrent synchronized includes both concurrent and coincident timing; serial includes sequential and expectant timing; mixed refers to tasks that are synchronous or serial in nature, depending on the need, suggesting expectant timing and - to a degree concurrent timing; and unsynchronized reflects asynchronous timing.

Considered together with dependence, one can posit that different action timings are better supported by a particular asymmetry [49]. While CAVR is intended as a conceptual framework supporting researchers, the alignment of timing and dependence may be additionally useful to designers of asymmetric VR. Figure 1 shows a reinterpreted version of the taxonomy initially presented by Thomsen et al. [49], designed to better reflect the logical tie between dependence, timing, and asymmetry. While the present work generally agrees with their interpretation of interaction patterns, Thomsen et al. [49:24] argue that the dependence in conditions of medium asymmetry is unidirectional "since both participants are dependent on each other, but in different ways." As this is the exact definition for bidirectional dependence given by Harris et al. [22], Figure 1 updates their organization of dependency and timing.

By adding a dimension related to the directionality of dependence, then, Thomsen and colleagues [49] posit that the asymmetry level can be attributed per situation. In Figure 1, high asymmetry refers to situations where one actor is uniquely dependent on another. Highly asymmetric systems are most supportive of work on separate tasks that may or may not require

PACM on Human-Computer Interaction, Vol. 5, No. CSCW1, Article 5, Publication date: April 2021. 
coordination because actors must communicate verbally to convey information due to the lack of a shared space. Low asymmetry occurs in situations where actors depend on one another in similar ways. In low asymmetry systems, each actor can communicate and interact with the virtual space as they work sequentially or together on tasks over an unspecified duration.

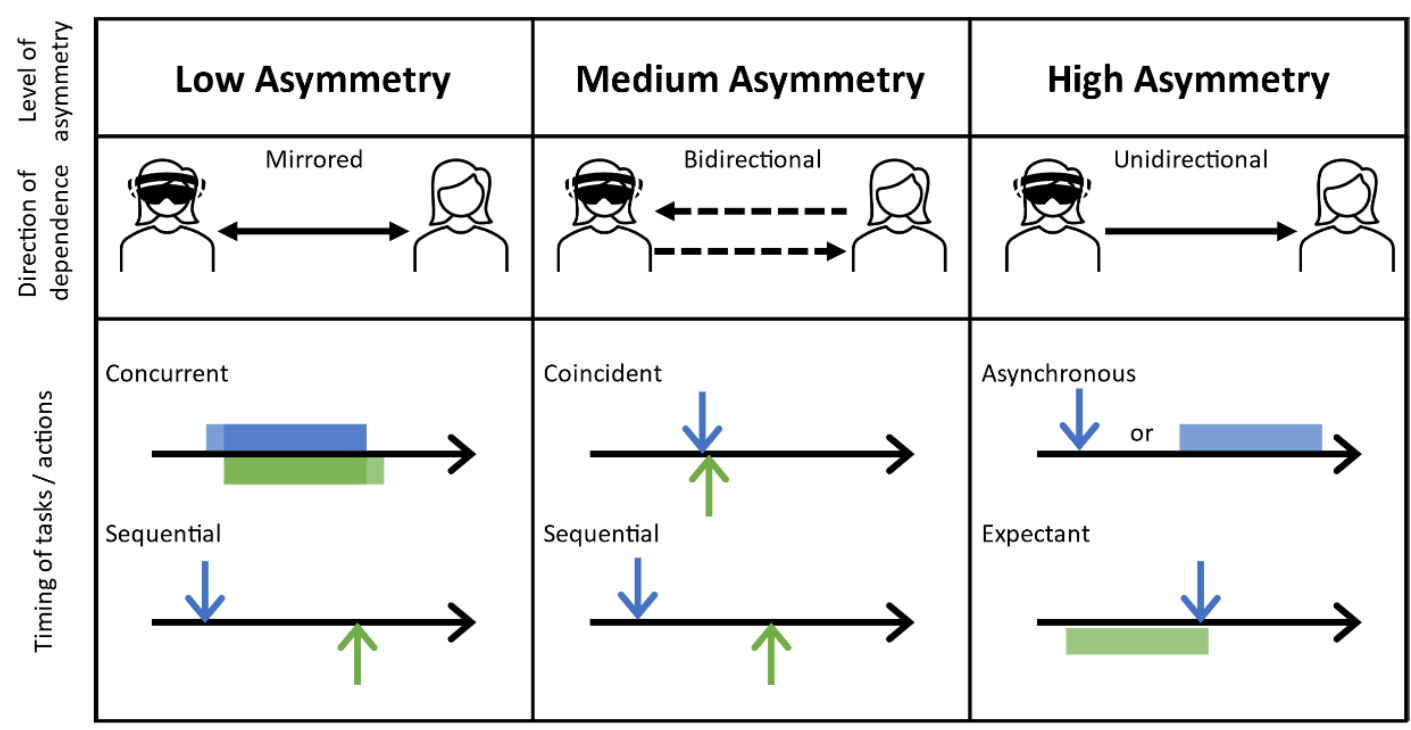

Figure 1. Organization of dependency and timing per asymmetry level; a new explanation of terms, integrating previous work [22,49]. Green bars (continuous tasks) and arrows (discrete tasks) on the bottom of the timeline-arrows show the HMD-user's tasks, while the blue demarcations on top of the same line indicate the same task types for the non-HMD user. Essentially, with low asymmetry, more synchronous, collaborative actions are feasible, while with high asymmetry, actions are typically asynchronous and expectant.

Whereas low and high asymmetry foster dependence, medium asymmetry is uniquely suited for positive interdependence, just as is typical for collaborative teams. Medium asymmetry features a digital interface that can transfer information between actors as they work on tasks that coincide or occur in a non-time-sensitive sequence. As such, interface and perceptual access decisions can create or alleviate friction in user experiences of mid-level asymmetric VR. Workspace awareness information in medium asymmetrical configurations may therefore be communicated through an additional interface rather than through the shared space or partner communication.

\section{CAVR: THE COMPOSITE FRAMEWORK FOR ASYMETRIC VR}

Theories existing to date explore facets of interest to co-located asymmetric VR system use, but no single model addresses the whole concept. CAVR (Table 2) extends the current conceptualization of asymmetry to encompass group dynamics in addition to interaction and technology characteristics by defining the dimensions of asymmetry. These five dimensions (spatial copresence, transportation, informational richness, team interdependence, and balance of power) draw from a restructuring of pre-existing definitions of asymmetry and an examination of the literature, and they are summarized below. 
Table 2. The Composite framework for Asymmetric VR (CAVR).

\begin{tabular}{cc}
$\begin{array}{c}\text { Asymmetry } \\
\text { Dimensions }\end{array}$ & $\begin{array}{c}\text { Definition } \\
\text { Spatial Co-presence }\end{array}$ \\
Transportation & $\begin{array}{c}\text { Group members receive different levels of mediated access to each } \\
\text { other; range of unmediated to mediated spatial co-presence } \\
\text { For each member, the mixed-reality space is reached through } \\
\text { different interaction metaphors supported by the technology }\end{array}$ \\
$\begin{array}{c}\text { Informational Richness } \\
\text { Team Interdependence } \\
\text { Balance of Power }\end{array}$ & $\begin{array}{r}\text { The extent to which the technology delivers and captures information } \\
\text { about the mixed-reality space }\end{array}$ \\
& $\begin{array}{c}\text { Group members bring different goals that align to various degrees } \\
\text { Grombers have different degrees of control over information, } \\
\text { influenced by their roles and levels of experience }\end{array}$ \\
\hline
\end{tabular}

1: adapted from [30], 2: adapted from [37], 3: adapted from [28]; 4: adapted from [3]. Spatial co-presence is a new blend of terms.

To appreciate the challenge of what an asymmetric VR system is trying to accomplish, it is worth noting that there are two worlds that team members must be able to engage: the real world in which they are co-located, which may contain physical objects with which they would like to interact, and the virtual world, which may also contain information and virtual objects with which they would like to interact. Essentially, the system, if well-designed, could offer the sense of engaging with both worlds simultaneously - not quite being in two places at once, but allowing relevant engagement with information, objects, and people in both worlds. In this paper the authors refer this overlapping space of the two worlds as the "mixed-reality space." This section introduces the five dimensions of CAVR and ties them to the 18 framework papers, noting how the dimensions emerged.

\subsubsection{Spatial Co-Presence}

Early research in the area of technology asymmetry focuses on the use of telepresence systems. Specifically, Kraus and Kibsgaard [30] note that the use of telepresence technology in face-to-face situations was not easily categorized using existing models of communication and interaction in VR. Extending pre-existing frameworks (including the virtuality continuum [39]), Kraus and Kibsgaard identified five dimensions of human-to-human communication during the use of immersive environments: communication channel; mediation of communication; and three communication situations - co-presence, transportation (see [3]), and virtuality (see [39]). Whereas co-presence in other literature refers to the feeling of experiencing a specific environment with another person [41], Kraus and Kibsgaard conceptualize co-presence as the result of the design decision to either mediate or not mediate the experience of co-presence [30]. In their view, co-presence seeks to mirror the experience in the real-world, rather than in the virtual space.

Interestingly, asymmetric VR is neither named nor entirely represented by Kraus and Kibsgaard [30]. For instance, while transportation indicates the degree of a person's separation from others in their physical location, the authors do not explore the area of mixed-presence (associated with the mixed-reality space) beyond the identification of "vicarious-transport situations," which use "look-into" as opposed to "step-into" as an interaction metaphor. 
This characterization reflects only part of the communication landscape in asymmetric VR, as a third interaction metaphor describes the interaction of two individual users with a shared object without any transportation into a shared space [43]. While first introduced to describe participation with symmetric collaborative virtual environments, the definition of "reach-into" interactions can be extended to describe asymmetric VR. Here, "reach-into" interactions give an outside perspective while also allowing the non-immersed user to interact with the virtual environment, thus CAVR extends Kraus and Kibsgaard's conceptualization of co-presence to instead focus on the mediation of the mixed-reality space. As such, CAVR integrates the ideas of Kraus and Kibsgaard [30], Otto, Roberts, and Wolff [43], and Benford and colleagues [3] to introduce the dimension of spatial co-presence or the degree of mediated access to the mixedreality space received by group members.

Spatial co-presence concerns the feeling of being with others in the mixed-reality space. In CAVR, the focus of co-presence turns from the feeling of being in a real space together to being in the mixed-reality space with one another. In the design team example from Section 1, the desktop and mouse/keyboard user will feel a lower level of togetherness with the other person in the mixed-reality space, despite sharing an office space (and thus experiencing a high co-presence in the physical space).

\subsubsection{Transportation}

From another perspective, asymmetric co-located VR is a mixed-reality-bound shared space, with the levels of asymmetry being dependent on various technology variables; according to Benford et al., these variables are transportation, artificiality, and spatiality [3]. Whereas the virtuality continuum focuses on the degree to which the information is digital (virtual) or real [39], Benford and colleagues' conceptualization recognizes that in addition to the realness of the space (artificiality), virtual spaces also vary in the extent to which they exclude the physical location (transportation) and the degree to which they create a new shared space (spatiality) [3]. As such, their conceptualization allows for coordinated use of XR technology.

When considering asymmetric VR experiences, the design must overcome many of the same barriers in distributed technology use even when users are in the same physical location. Because HMDs occlude the user's vision, non-HMD-wearing users of the same physicality effectively occupy a different space [30]. Therefore, despite being co-located and synchronous (and thus outside of the traditional context of telepresence [2,3,30]), both categories of individuals need to access the virtual environment and interact with one another during coordinated action.

In other words, while the HMD-user is immersed in the task space (here, the virtual environment), they do not have access to the person space (due to HMD-triggered occlusion) $[2,10]$. Conversely, the external users have access to the person space, meaning they can make sense of the emotions, personality, and identity of the HMD-user, without having access to task space. Asymmetric VR introduces a shared reference space (the mixed-reality space), improving user presence by contextualizing the person space's information within the task space $[2,10]$. As such, asymmetric VR is responsible for creating a shared space, allowing the transportation of both immersed and external users into the shared virtual environment while maintaining awareness of relevant features of the real-world environment. CAVR's definition of transportation, then, can be understood as the differences in the attainability of the mixed-reality space, depending on the type of technology used by each group member.

Transportation is related to co-presence, yet it diverges due to its focus on a user's actual interaction in either a new, virtual environment or their original environment, rather than the 
perception of interacting in a new place [3]. Systems with varying transportation levels, from high transportation systems like VR HMDs to the lower-level transportation AR devices, may cater to different tasks, which would benefit the heterogeneous groups common to coordinated action [32]. Transportation does include the hardware and software aspects of user interface, and could thus be deemed an objective, measurable dimension, but it differs from the classic definition of VR immersion [8] in that transportation focuses more on the degree to which you can access the affordances of both the virtual and real world rather than the fidelity of your virtual world experience. As such, a designer wearing an HMD to test a virtual design would experience a "stepinto" level of transportation, while the user of the desktop and keyboard/mouse would experience a "reach-into" level of transportation, assuming the application has been designed to allow realtime interaction with the mixed-reality space (either through the interface or through information transfer with the HMD-user).

\subsubsection{Team Interdependence}

In organizational psychology, the pattern or extent of interaction required for task completion is termed "interdependence" [4]. Whereas Harris and Hancock [21] identify interdependence as a complementary - yet separate - concept to asymmetry, CAVR incorporates team interdependence, or the degree of alignment between group member goals, as a dimension of asymmetry. Interdependence is a concept that is inextricably linked to the definition of a team, differentiating teamwork from group work [4], which only requires the dependence of group members. Dependence, then, describes the two-dimensional concept [44] that includes mutuality (or, in Harris [21,22] terms, direction) and level (which is related to timing).

Team interdependence arises within groups whose members must rely on one-another to accomplish their shared goals [21]. The team composition can play into this need, as a team with varied roles will need to integrate the work of its members to achieve its goals [11]. Lee and Paine's [32] Model of Coordinated Action (MoCA) dimension of "Number of Communities of Practice" describes the heterogeneity of a group joined in an instance of coordinated action. Each additional community of practice introduces different roles, norms, tools, and expectations [31]. These heterogeneous groups consist of members who bring a different set of goals that may or may not align well with the group, representing higher or lower levels of team interdependence. Within the Section 1 design team example, the HMD user seeks to evaluate the alignment between the experience and the intentions of the application, while the desktop user takes note of these issues and adjusts the design to increase alignment. These goals represent a relatively high level of alignment, and thus the group experiences a high level of team interdependence, rather than mutual or unidirectional dependence.

\subsubsection{Balance of Power}

In their work with mixed-reality boundaries, Benford et al. [3] warn that these spaces' mutuality is critical to the balance of power, a term that is adopted here. In CAVR, this is defined as the degrees of access to and control of information in an asymmetric or mixed-reality space, and it is determined by the members' roles (including software permissions), access to hardware, and levels of experience. While some of this information comes from the individual's prior experience, or priors, as in Carlile's boundaries framework [11], the boundary object (e.g., the interface used alongside VR) is responsible for providing a shared language for conveying knowledge and understanding differences (such as hidden information [46]) in order to understand something more deeply [10].

PACM on Human-Computer Interaction, Vol. 5, No. CSCW1, Article 5, Publication date: April 2021. 
The balance of power within a group is dependent of the levels of interdependence and the mutuality of the boundaries to the mixed-reality space generated during asymmetric VR experiences. Under conditions of high team interdependence, power flows between group members such that it remains balanced between them. The boundaries between the physical and virtual spaces have been previously examined as ways to improve the understanding of the goings-on in those spaces [3]. Using boundary objects, which are objects designed to share information across contexts [11], Benford and colleagues allowed users to communicate workspace information across these boundaries [3]. When using asymmetric VR, differences in the mutuality of the mixed-reality boundaries [3] or the quality of the information provided by the boundary object [11] can change the group's proportions of dominance and control. For example, when considering a design team crafting a virtual experience, using the screen-mirroring function (a "look-into" metaphor) available to most VR applications would result in higher dominance over the mixed-reality space for the HMD-user. However, as soon as the external user (here, the desktop user) is given access to a different level of transportation, spatial co-presence, or informational richness (described next), the balance of power shifts in their favor.

\subsubsection{Informational Richness}

Another area in which asymmetric VR falls through gaps in existing conceptual frameworks is in the literature surrounding virtual teams. Virtual teams are commonly understood as being distributed and requiring technology to interact with one another [28,37], yet teams now incorporate technology in ways that enhance their coordinated action [42]. These technologies vary in their informational richness levels, a term introduced by Kirkman and Mathieu [28] to underscore the differences in technologies' media richness [29], specifically in terms of information valuable to a team. Indeed, this conceptualization is relevant to CAVR, which extends the focus of extant asymmetry definitions to include the distribution of team-relevant details such as workspace awareness information. CAVR's dimension of informational richness concerns the extent to which the technology delivers and captures information about the mixed-reality space.

Informational richness is related to the quality of the boundary object and is pivotal to the communication effectiveness of a team $[11,19,37]$. Especially in teams with multiple specialized roles, the sharing of knowledge across the boundaries to understanding that are introduced by the coming-together of various communities of practice is critical to success [11]. The same is true for the sharing of information across the boundaries of the mixed-reality space. If the design team used a motion-tracked touchscreen to allow the external user of the mixed-reality design space to place notes in the space, rather than just intermingling screenshots with text in a text document ("reach-into"), informational richness would be high. Informational richness could also be high for a desktop user if the goal is to make live edits to a virtual environment. Higher levels of informational richness occur when the interaction metaphor (between "reach-into" and "lookinto") supplies the information that is necessary to the team's function. Therefore, in addition to interaction and balance of power differences, the boundary object's quality also affects the informational richness for each group member.

\section{DISCUSSION AND CONCLUSIONS}

By combining the ideas from video games and $\mathrm{CSCW}$ - including the ideas from MoCA, media spaces, and asymmetric video game design, it is intended that the CAVR will better describe the 
real state of asymmetric VR use in co-located groups. Whereas the Thomsen et al. [49] taxonomy included information about how the levels of asymmetry would relate to the timing or synchronicity responsible for facilitating effective interaction, CAVR will aid researchers in understanding how asymmetry affects the group dynamics and other related variables. Additionally, while their taxonomy was established with a focus on collaborative learning, the present framework is expected to interface more directly with models in CSCW, extending them to include asymmetric workspace interface considerations.

When examined in context, a system's asymmetry is expected to impact its users' experiences and behaviors. Within medium asymmetry environments, such as the use of an HMD and desktop application in a design team, workspace awareness cues will be most beneficial to the members. When the members experience different transportation and spatial co-presence levels, these cues must be mediated by the technology. As described in previous work [30], establishing explicit mediation through the boundary object (such as a smartphone app or a desktop interface that allows interaction across the mixed-reality boundaries) is associated with a high cost-to-benefit ratio. Through that boundary object, informational richness and spatial co-presence can vary to meet the needs of each member's tasks.

For asymmetric VR use, the technology may facilitate or encourage interaction as an enhancement technology. Briefly explored in Section 1.1, teamwork dynamics and composition are expected to influence the choice of asymmetric VR system. In line with research in teamwork [54], the team composition is also likely to affect the teamwork processes and emergent states and, in turn, the performance outcomes. As such, heterogeneous groups (i.e., those with a varied team composition) using VR may benefit from the asymmetry-supported team interdependence (an emergent state in team theory) and informational richness of asymmetric configurations. While the dimensions of CAVR could be used to formulate symmetric answers to a group's needs, asymmetry can invoke team interdependence [21] and should not be overlooked as a challenge to be "righted" by design. The present framework lays a path for future research into how collaboration in the mixed-reality space formed by asymmetric VR affects groups and group functions.

While the call for more financially accessible technology has been raised, attention to other areas of digital accessibility, including design guidance, has been lacking. In asymmetric VR, support for individual differences in preference and visually induced motion sickness tolerance may be designed into a personalized and even adaptive experience via asymmetric game design. By researching design strategies for more inclusive experiences, the path toward reaching more diverse audiences becomes clearer, moving VR in a more accessible direction, beyond just being accessible in terms of cost. In addition, it is critical to establish a common language for describing asymmetry, which will continue to exist even as the technology gets cheaper and easier to use [34,53].

Perhaps surprisingly, the link between asymmetric VR and team processes is not well established. While organizational psychology theories were deemed relevant to the scoping review, they were only mentioned in one paper, which was published recently (see Harris and Hancock's 2019 work [21]). In games and in life, asymmetries can make an experience engaging, and the ways different people use the different abilities they have, whether augmented by technology or not, can drastically alter the outcomes of any collaboration. For a technology that can have a meaningful impact on teamwork in organizations, broadly, more attention to the findings and recommendations of work from organizational psychology is needed. 


\subsection{Future Work}

This paper signifies the first step toward the creation of CAVR, the Composite framework for Asymmetric VR, formed by gathering relevant theories, concepts, and models. The next step for this research is to evaluate the apparent relationships between CAVR and related areas, such as user experience design (specifically for asymmetric VR) and team outcomes. Ideally, these evaluative efforts will foster the use of CAVR in the design of asymmetric experiences. Further, the affordances of asymmetric VR and the actions they support should be categorized. Perhaps a set of scales for each dimension should be generated so that CAVR can be used to compare two asymmetric VR systems numerically. Currently, there exists a categorization of actions relevant to gaming [22,49], yet domains beyond ludology have not been tapped in this way.

While the present work delineates an interpretation of the various possible dimensions of asymmetry, there are still unknowns about the relationship between these dimensions and the experiences they invoke. While Thomsen and colleagues [49] describe how the game design mechanics of asymmetry [22] can be mapped to actual VR gameplay through the kinds of dependence and timing they encourage, their taxonomy is yet untested. Similarly, we suggest that the Framework of Enhancement Technology [42] could be used to specify the interaction role of an asymmetric VR experience. Perhaps an artifact of the lack of empirical study of the area, the boundaries between the levels of asymmetry are not clearly defined. Indeed, we consider the evaluation of CAVR a priority for future research.

Finally, future work should explore how team performance and communication outcomes relate to CAVR's conceptualization of asymmetric VR. In doing so, research can guide the creation of technology that seeks to work with the existing differences among us, rather than fight them. Such technology would be an optimal collaboration tool for coordinated action in distributed and co-located teams.

\section{ACKNOWLEDGMENTS}

The authors thank Carsten Scherber and Emily Oldham for their contributions to the dimensions of CAVR as well as Dr. Larysa Nadolny and Mariangely Iglesias Pena for their insight during the formulation of the paper. Additionally, we are grateful for the suggestions raised by our reviewers, without whom the current version of this work would not be possible.

\section{REFERENCES}

[1] Kenneth J. Arrow. 1963. Uncertainty and the Welfare Economics of Medical Care. Am. Econ. Rev.53, 5 (1963), $941-$ 973.

[2] Ron Baecker, Steve Harrison, Bill Buxton, Steven Poltrock, and Elizabeth Churchill. 2008. Media spaces: Past visions, current realities, future promise. In Proceeding of the twenty-sixth annual CHI conference extended abstracts on Human factors in computing systems - CHI '08, Association for Computing Machinery, Florence, Italy, 2245-2248. DOI: https://doi.org/10.1145/1358628.1358660

[3] Steve Benford, Chris Greenhalgh, Gail Reynard, Chris Brown, and Boriana Koleva. 1998. Understanding and constructing shared spaces with mixed-reality boundaries. ACM Trans. Comput. Interact.5, 3 (September 1998), 185223. DOI:https://doi.org/10.1145/292834.292836.

[4] Lauren E Benishek and Elizabeth H Lazzara. 2019. Teams in a New Era: Some Considerations and Implications. Front. Psychol.10, 1006 (2019), 1-15. DOI:https://doi.org/10.3389/fpsyg.2019.01006.

[5] Anastasiia Beznosyk, Peter Quax, Wim Lamotte, and Karin Coninx. 2012. The Effect of Closely-Coupled Interaction on Player Experience in Casual Games. In Entertainment Computing-ICEC 2012, Springer Berlin Heidelberg, 243255. Retrieved from www.soe.com

[6] Walter R Boot, Andrew Dilanchian, and Ronald Andringa. 2019. Exploring Older Adults' Perceptions of Presence and Immersion in Diverse Virtual Environments. Innov. Aging 3, Supplement_1 (November 2019), S239-S240. DOI:https://doi.org/10.1093/geroni/igz038.895. 
[7] Matt Bower, Mark J.W. Lee, and Barney Dalgarno. 2017. Collaborative learning across physical and virtual worlds: Factors supporting and constraining learners in a blended reality environment. Br. J. Educ. Technol.48, 2 (March 2017), 407-430. DOI:https://doi.org/10.1111/bjet.12435.

[8] Doug A. Bowman and Ryan P. McMahan. 2007. Virtual Reality: How Much Immersion Is Enough? Computer (Long. Beach. Calif).40, 7 (July 2007), 36-43. DOI:https://doi.org/10.1109/MC.2007.257.

[9] Jon Ram Bruun-Pedersen, Stefania Serafin, and Lise Busk Kofoed. 2016. Going Outside While Staying Inside Exercise Motivation with Immersive vs. Non-immersive Recreational Virtual Environment Augmentation for Older Adult Nursing Home Residents. In2016 IEEE International Conference on Healthcare Informatics (ICHI), IEEE, Chicago, IL, 216-226. DOI:https://doi.org/10.1109/ICHI.2016.31.

[10] Bill Buxton. 2009. Mediaspace - Meaningspace - Meetingspace. In Media Space $20+$ Years of Mediated Life, Steve Harrison (ed.). Springer, London, 217-231. DOI:https://doi.org/10.1007/978-1-84882-483-6_13

[11] Paul R. Carlile. 2002. A pragmatic view of knowledge and boundaries: Boundary objects in new product development. Organ. Sci.13, 4 (2002), 442-456. DOI:https://doi.org/10.1287/orsc.13.4.442.2953.

[12] Marco Cavallo, Mishal Dolakia, Matous Havlena, Kenneth Ocheltree, and Mark Podlaseck. 2019. Immersive Insights: A Hybrid Analytics System for Collaborative Exploratory Data Analysis. In25th ACM Symposium on Virtual Reality Software and Technology (VRST '19), Association for Computing Machinery, Parramatta, NSW, Australia, 1-12. DOI:https://doi.org/10.1145/3359996.3364242.

[13] Alan Dix, Janet Finlay, Gregory D. Abowd, and Russell Beale. 2004. Design Rules. In Human-Computer Interaction (Third Edit). Pearson Education limited.

[14] Barrett Ens, Joel Lanir, Anthony Tang, Scott Bateman, Gun Lee, Thammathip Piumsomboon, and Mark Billinghurst. 2019. Revisiting collaboration through mixed reality: The evolution of groupware. Int. J. Hum. Comput. Stud.131, February (2019), 81-98. DOI:https://doi.org/10.1016/j.ijhcs.2019.05.011.

[15] Juliano Franz, Mohammed Alnusayri, Joseph Malloch, and Derek Reilly. 2019. A Comparative Evaluation of Techniques for Sharing AR Experiences in Museums. Proc. ACM Human-Computer Interact.3, CSCW (November 2019), 124:1-20. DOI:https://doi.org/10.1145/3359226.

[16] Jeronimo Gustavo Grandi, Henrique Galvan Debarba, and Anderson Maciel. 2019. Characterizing Asymmetric Collaborative Interactions in Virtual and Augmented Realities. In IEEE Conference on Virtual Reality and 3D User Interfaces (IEEEVR 2019), IEEE, 127-135. DOI:https://doi.org/10.1109/vr.2019.8798080.

[17] Jan Gugenheimer, Mark McGill, Frank Steinicke, Christian Mai, Julie Williamson, and Ken Perlin. 2019. Challenges using head-mounted displays in shared and social spaces. In Conference on Human Factors in Computing Systems Proceedings, 1-8. DOI:https://doi.org/10.1145/3290607.3299028.

[18] Jan Gugenheimer, Evgeny Stemasov, Julian Frommel, and Enrico Rukzio. 2017. ShareVR: Enabling Co-Located Experiences for Virtual Reality between HMD and Non-HMD Users. In Proceedings of the 2017 CHI Conference on Human Factors in Computing Systems, Association for Computing Machinery, Denver, CO, USA, 4021-4033. DOI:https://doi.org/10.1145/3025453.3025683.

[19] Carl Gutwin and Saul Greenberg. 2002. A Descriptive Framework of Workspace Awareness for Real-Time Groupware. Comput. Support. Coop. Work 11, (September 2002), 411-446. DOI:https://doi.org/10.1023/A:1021271517844

[20] P.J. Hammer, D. Haas-Wilson, W.M. Sage, and M.A. Peterson (Eds.). 2003. Uncertain times: Kenneth Arrow and the changing economics of health care. Duke University Press.

[21] John Harris and Mark Hancock. 2019. To Asymmetry and Beyond! In Proceedings of the 2019 CHI Conference on Human Factors in Computing Systems - CHI '19, ACM, Glasgow, Scotland UK, 1-12. DOI:https://doi.org/10.1145/3290605.3300239

[22] John Harris, Mark Hancock, and Stacey D. Scott. 2016. Leveraging Asymmetries in Multiplayer Games. In Proceedings of the 2016 Annual Symposium on Computer-Human Interaction in Play Companion Extended Abstracts - CHI PLAY Companion '16, ACM, Austin, TX, USA, 350-361. DOI:https://doi.org/10.1145/2967934.2968113

[23] Christian Heath and Paul Luff. 1992. Media Space and Communicative Asymmetries: Preliminary Observations of Video-Mediated Interaction. Human-Computer Interact.7, 3 (September 1992), 315-346. DOI:https://doi.org/10.1207/s15327051hci0703_3

[24] Rorik Henrikson, Bruno De Araujo, Fanny Chevalier, Karan Singh, and Ravin Balakrishnan. 2016. Multi-device storyboards for cinematic narratives in VR. UIST 2016 - Proc. 29th Annu. Symp. User Interface Softw. Technol. (2016), 787-796. DOI:https://doi.org/10.1145/2984511.2984539.

[25] Robin Hunicke, Marc Leblanc, and Robert Zubek. 2004. MDA: A formal approach to game design and game research. AAAI Work. - Tech. Rep.WS-04-04, (2004), 1-5.

[26] Robert Johansen. 1988. Groupware computer support for business teams. The Free Press.

[27] David W. Johnson and Roger T. Johnson. 1994. Constructive Conflict in the Schools. J. Soc. Issues 50, 1 (1994), 117137. DOI:https://doi.org/10.1111/j.1540-4560.1994.tb02401.x 
[28] Bradley L Kirkman and John E Mathieu. 2005. The Dimensions and Antecedents of Team Virtuality. J. Manage.31, (2005), 700-718. DOI:https://doi.org/10.1177/0149206305279113

[29] Ned Kock. 1998. Can communication medium limitations foster better group outcomes? An action research study. Inf. Manag.34, (1998), 295-305.

[30] Martin Kraus and Martin Kibsgaard. 2015. A Classification of Human-to-Human Communication during the Use of Immersive Teleoperation Interfaces. In Proceedings of the 2015 Virtual Reality International Conference on ZZZ VRIC '15, ACM Press, New York, New York, USA, 1-8. DOI:https://doi.org/10.1145/2806173.2806198

[31] J. Lave and E. Wenger. 1991. Situated learning: Legitimate peripheral participation. Cambridge University Press.

[32] Charlotte P. Lee and Drew Paine. 2015. From the matrix to a model of coordinated action (MoCA): A conceptual framework of and for CSCW. In Proceedings of the 18th ACM Conference on Computer Supported Cooperative Work \& Social Computing - CSCW '15, ACM Press, Vancouver, BC, Can, 179-194. DOI:https://doi.org/10.1145/2675133.2675161

[33] Paul Luff, Christian Heath, and David Greatbatch. 1992. Tasks-in-interaction: Paper and screen based documentation in collaborative activity. In Computer Supported Cooperative Work (CSCW), 163-170. DOI:https://doi.org/10.1145/143457.143475

[34] Paul Luff, Christian Heath, Hideaki Kuzuoka, Jon Hindmarsh, Keiichi Yamazaki, and Shinya Oyama. 2003. Fractured Ecologies: Creating Environments for Collaboration. Human-Computer Interact.18, 1-2 (June 2003), 51-84. DOI:https://doi.org/10.1207/S15327051HCI1812_3

[35] Paul Luff, Christian Heath, Menisha Patel, Dirk Vom Lehn, and Andrew Highfield. 2018. Creating Interdependencies: Managing Incidents in Large Organizational Environments. Human-Computer Interact.33, 5-6 (2018), 544-584. DOI:https://doi.org/10.1080/07370024.2017.1412830

[36] Paul K. Luff, Naomi Yamashita, Hideaki Kuzuoka, and Christian Heath. 2015. Flexible ecologies and incongruent locations. Conf. Hum. Factors Comput. Syst. - Proc.2015-April, (2015), 877-886. DOI:https://doi.org/10.1145/2702123.2702286

[37] Shannon L. Marlow, Christina N. Lacerenza, and Eduardo Salas. 2016. Communication in virtual teams: a conceptual framework and research agenda. Hum. Resour. Manag. Rev.27, 4 (2016), 575-589. DOI:https://doi.org/10.1016/j.hrmr.2016.12.005

[38] Mark McGill, Daniel Boland, Roderick Murray-Smith, and Stephen Brewster. 2015. A Dose of Reality: Overcoming Usability Challenges in VR Head-Mounted Displays. In Proceedings of the 33rd Annual ACM Conference on Human Factors in Computing Systems - CHI '15, ACM Press, Seoul, S Korea, 2143-2152. DOI:https://doi.org/10.1126/science.346.6212.908

[39] Paul Milgram and Fumio Kishino. 1994. Taxonomy of mixed reality visual displays. IEICE Trans. Inf. Syst.E77-D, 12 (1994), 1321-1329.

[40] Zachary Munn, Micah D.J. Peters, Cindy Stern, Catalin Tufanaru, Alexa McArthur, and Edoardo Aromataris. 2018. Systematic review or scoping review? Guidance for authors when choosing between a systematic or scoping review approach. BMC Med. Res. Methodol.18, 1 (2018), 1-7. DOI:https://doi.org/10.1186/s12874-018-0611-x

[41] Catherine S. Oh, Jeremy N. Bailenson, and Gregory F. Welch. 2018. A systematic review of social presence: Definition, antecedents, and implications. Front. Robot. AI 5, OCT (2018), 1-35. DOI:https://doi.org/10.3389/frobt.2018.00114

[42] Thomas Olsson, Pradthana Jarusriboonchai, Paweł Woźniak, Susanna Paasovaara, Kaisa Väänänen, and Andrés Lucero. 2019. Technologies for Enhancing Collocated Social Interaction: Review of Design Solutions and Approaches. Comput. Support. Coop. Work (February 2019). DOI:https://doi.org/10.1007/s10606-019-09345-0

[43] Oliver Otto, Dave Roberts, and Robin Wolff. 2006. A review on effective closely-coupled collaboration using immersive CVE's. Proc. - VRCIA 2006 ACM Int. Conf. Virtual Real. Contin. its Appl. (2006), 145-154. DOI:https://doi.org/10.1145/1128923.1128947

[44] Christopher Poile. 2010. Asymmetric dependence and its effect on helping behaviour. University of Waterloo. Retrieved from https:/uwspace.uwaterloo.ca/bitstream/handle/10012/4954/Poile_Christopher.pdf

[45] Roy A. Ruddle, Justin C. D. Savage, and Dylan M. Jones. 2002. Symmetric and asymmetric action integration during cooperative object manipulation in virtual environments. ACM Trans. Comput. Interact.9, 4 (December 2002), 285308. DOI:https://doi.org/10.1145/586081.586084

[46] Serena G. Sohrab, Mary J. Waller, and Seth Kaplan. 2015. Exploring the Hidden-Profile Paradigm: A Literature Review and Analysis. Small Gr. Res.46, 5 (2015), 489-535. DOI:https://doi.org/10.1177/1046496415599068

[47] Anthony Steed, William Steptoe, Wole Oyekoya, Fabrizio Pece, Tim Weyrich, Jan Kautz, Doron Friedman, Angelika Peer, Massimiliano Solazzi, Franco Tecchia, Massimo Bergamasco, and Mel Slater. 2012. Beaming: An asymmetric telepresence system. IEEE Comput. Graph. Appl.32, 6 (2012), 10-17. DOI:https://doi.org/10.1109/MCG.2012.110

[48] Steel Crate Games. 2015. Keep Talking and Nobody Explodes. Retrieved from https://store.steampowered.com/app/341800/Keep_Talking_and_Nobody_Explodes/ 
[49] Lui Albæk Thomsen, Niels Christian Nilsson, Rolf Nordahl, and Boris Lohmann. 2019. Asymmetric collaboration in virtual reality. Tidsskr. Læring og Medier 12, 20 (March 2019), 1-28. DOI:https://doi.org/10.7146/lom.v12i20.109391

[50] Güliz Tokaldi, Kaitlyn Ouverson, Chase Meusel, Austin Garcia, Stephen B. Gilbert, and Michael C. Dorneich. 2018. An Analysis of Video Games Using the Dimensions of Human-Agent Interaction. In Proceedings of the Human Factors \& Ergonomics Society (HFES) Annual Meeting, 716-720.

[51] Amy Voida, Stephen Voida, Saul Greenberg, and Helen Ai He. 2008. Asymmetry in media spaces. Proc. ACM Conf. Comput. Support. Coop. Work. CSCW (2008), 313-322. DOI:https://doi.org/10.1145/1460563.1460615

[52] WalkinVR. 2020. WalkinVR Driver. Retrieved from https://walkinvrdriver.com

[53] Erin Casey Whitworth. 2017. Hetero-Technic Cooperation with Computing and Non-Computing Technologies: A Study of the Transmodal Capacity of Prosodic Cues to Alleviate Asymmetric Access to Tactile Phenomena. The University of Texas at Austin. Retrieved from https://repositories.lib.utexas.edu/handle/2152/63565

[54] Christopher W. Wiese, Marissa L. Shuffler, and Eduardo Salas. 2015. Teamwork and Team Performance Measurement (Second Edi ed.). Elsevier. DOI:https://doi.org/10.1016/B978-0-08-097086-8.22017-5

[55] Jason Wuertz, Sultan A. Alharthi, William A. Hamilton, Scott Bateman, Carl Gutwin, Anthony Tang, Zachary O. Toups, and Jessica Hammer. 2018. A design framework for awareness cues in distributed multiplayer games. Conf. Hum. Factors Comput. Syst. - Proc.2018-April, (2018), 1-14. DOI:https://doi.org/10.1145/3173574.3173817

Received October 2020; revised January 2021; accepted January 2021. 